\title{
Suggestions for clinical study of exercise rehabilitation
}

\author{
Chang-Ju Kim (iD https://orcid.org/0000-0003-4749-5795
}

In this issue (Vol.: 14, No.: 5, 2018) of the Journal of Exercise Rebabilitation, articles on exercise rehabilitation were published from many countries and in various fields. The study on the mechanism of exercise rehabilitation through animal experiments, the study of psychological factors with rehabilitation, and the effects of exercise rehabilitation for various diseases have been extensively discussed in this issue.

In the animal studies, the effect of type II collagen extract on immunosuppression, the effect of exercise on epileptogenic rats, and the effect of exercise on stress-inducing animals were published.

In the psychological fields, the study on the difference in humanity about sports injuries, the effect of whole-body vibration on agility, quick reaction ability, and elasticity, the study on physical activity limitation in cancer patients, the effect of treadmill cognitive dual task gait training on the gait ability in chronic stroke, the study of association with the club's movement, and the study on declining cognition due to aging etc. were included.

The researches of exercise rehabilitation for various diseases included the effect of exercise and electrical stimulation on pain, the study on the relationship between food intake and immune function, the effect of exercise rehabilitation on muscular atrophy patients, the effect of exercise on breathing exercise, the effect of exercise on cardiopulmonary function, metabolic factors, and self-esteem of obese women, the effect of aquatic exercise on knee osteoarthritis, the study on the changes in muscle strength and balance during hearing and visual impairment, and the effect of $\mathrm{N}$-acetylcysteine on cell damage and oxidative stress etc.
In the Journal of Exercise Rebabilitation, the efficacy verification of exercise rehabilitation for diverse diseases is most frequently handled. However, clinical studies should consider both ethical issues and scientific evidence. First of all, clinical study is an experiment targeting human, it is necessary to observe the research ethics procedure. Clinical studies that identify the effects of exercise rehabilitation require a sufficient number of subjects to provide a scientific basis. Instead of simply presenting measurement results, it is necessary to use various techniques to derive the results and to present the mechanisms. Statistical interpretation will also be an important factor in the outcome judgment. Studies of specific diseases in the local region or country will be more meaningful.

Through these efforts, the Journal of Exercise Rebabilitation can be an integrated vehicle for finding ways to overcome modern diseases that are currently difficult to treat. The editorial board of the Journal of Exercise Rebabilitation gives thanks again to researchers from many countries who have contributed their articles.

\section{CONFLICT OF INTEREST}

No potential conflict of interest relevant to this article was reported.

President of the Korean Society of Exercise Rehabilitation Department of Physiology, College of Medicine Kyung Hee University, 26 Kyungheedae-ro, Dongdaemun-gu, Seoul 02447, Korea E-mail: changju@khu.ac.kr 